\title{
Predictive Factors of Recurrence of Papillary Thyroid Microcarcinomas: Analysis of 2,538 Patients
}

\author{
Andre de Ywata Carvalho ${ }^{1}$ (1) \\ Hugo Fontan Kohler ${ }^{1}$ Camila Couto Gomes $^{2}{ }^{(0)}$ \\ José Guilherme Vartanian ${ }^{10}$ \\ Luiz Paulo Kowalski,3 \\ 1 Head and Neck Surgery and Otorhinolaryngology Department, A. C. \\ Camargo Cancer Center, São Paulo, Brazil \\ 2 Surgical Oncology Division, A. C. Camargo Cancer Center, São Paulo, \\ Brazil \\ ${ }^{3}$ Head and Neck Surgery Department, Faculdade de Medicina, \\ Universidade de São Paulo, São Paulo, Brazil \\ Address for correspondence Andre Ywata de Carvalho, MD, MBA, \\ PHD, A. C. Camargo Cancer Center, Rua Professor Antonio Prudente \\ 211, 01509-010, São Paulo, Brazil \\ (e-mail: andreywata@bol.com.br; hkohler75@gmail.com; \\ camilacoutog@yahoo.com.br; jgvartanian@uol.com.br; \\ Ip_kowalski@uol.com.br).
}

Int Arch Otorhinolaryngol 2021;25(4):e585-e593.

\begin{abstract}
Introduction The incidence of papillary thyroid microcarcinoma (PTMC) has increased, and its treatment remains controversial.

Objective To identify the clinical and pathological factors predictive of tumor recurrence.

Methods We retrospectively analyzed 2,538 consecutive patients treated for PTMC, most submitted to total thyroidectomy (98\%) followed by radioactive iodine (RAI) ablation (51.7\%) at a cancer center from 1996 to 2015. The patients were stratified according to the American Thyroid Association (ATA) risk categories (low, intermediate, or high), and the clinicopathological features were evaluated by multivariate Cox regression analysis to identify independent prognostic factors for recurrence.

Results After a mean follow-up of 58 months (range: 3 to 236.5 months), tumor recurrence was diagnosed in 63 (2.5\%) patients, mostly in the lymph nodes. Distant metastasis occurred in $2(0.1 \%)$ patients. There were no cancer-related deaths. The multivariate analysis showed that age $<55$ years $(p=0.049$; hazard ratio [HR]: 2.54; $95 \%$ confidence interval [ $95 \% \mathrm{Cl}]: 0.95$ to 0.99$)$, multifocality $(p=0.032 ; \mathrm{HR}: 1.76 ; 95 \%$ $\mathrm{Cl}: 1.05$ to 2.96$)$, and the presence of lymph-node metastasis ( $p<0.001 ; \mathrm{HR}: 3.69 ; 95 \%$

Keywords

- thyroid

- papillary microcarcinoma

- recurrence

- prognosis

- risk stratification

$\mathrm{Cl}$ : 2.07-6.57) were independent risk factors for recurrence. Recurrence was observed in $29(1.5 \%)$ out of 1,940 low-risk patients, $32(5.4 \%)$ out of 590 intermediate-risk patients, and in 2 (25\%) out of 8 high-risk patients.

Conclusions The prognosis of PTMC is excellent, favoring a conservative treatment for most patients. Age $<55$ years, multifocality, and node metastasis at diagnosis, as well the ATA staging system effectively predict the risk of recurrence. The presence of these risk factors can help identify patients who should be considered for more aggressive management and more frequent follow-up.
\end{abstract}

received

June 15,2020

accepted

DOI https://doi.org/ 10.1055/s-0040-1722253. ISSN 1809-9777. (c) 2021. Fundação Otorrinolaringologia. All rights reserved.

This is an open access article published by Thieme under the terms of the Creative Commons Attribution-NonDerivative-NonCommercial-License, permitting copying and reproduction so long as the original work is given appropriate credit. Contents may not be used for commercial purposes, or adapted, remixed, transformed or built upon. (https://creativecommons.org/ licenses/by-nc-nd/4.0/)

Thieme Revinter Publicações Ltda., Rua do Matoso 170, Rio de Janeiro, RJ, CEP 20270-135, Brazil 


\section{Introduction}

The incidence of papillary thyroid carcinoma (PTC) is increasing worldwide ${ }^{1}$ mainly due to the greater number of tumors incidentally discovered after the widespread use of ultrasound-guided fine-needle aspiration (FNA) in patients with suspected thyroid diseases. Most of these tumors are papillary thyroid microcarcinomas (PTMCs), ${ }^{2-4}$ which are defined by the World Health Organization (WHO) as carcinomas $\leq 1 \mathrm{~cm}$ in the greatest dimension. ${ }^{5}$ Papillary thyroid microcarcinomas are less aggressive than PTCs $>1$ $\mathrm{cm}^{6}$ and most thyroid nodules $<1 \mathrm{~cm}$ should not undergo FNA.

Several previously-published series have demonstrated excellent outcomes following the therapy for PTMC, with a negligible cancer mortality rate. However, despite the favorable long-term prognosis, cases of locoregional recurrences and even distant metastases have been described. ${ }^{7,8}$

There is no consensus regarding the natural history of PTMC, and the treatment options range from observation to total thyroidectomy and neck lymph-node dissection followed by radioactive iodine (RAI) ablation. ${ }^{9}$ An active surveillance management approach to low-risk PTMC is a safe and effective alternative to immediate surgical resection. $^{10,11}$ The 2015 American Thyroid Association (ATA) guidelines state that "if surgery is chosen for patients with thyroid cancer $<1 \mathrm{~cm}$ without extrathyroidal extension and cN0, the initial surgical procedure should be a thyroid lobectomy, unless there are clear indications to remove the contralateral lobe. Thyroid lobectomy alone is sufficient treatment for small, unifocal, intrathyroidal carcinomas in the absence of prior head and neck radiation, familial thyroid carcinoma, or clinically-detectable cervical nodal metasta-

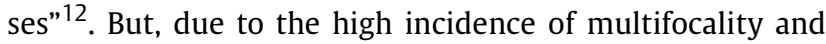
lymph node metastasis in level VI, some authors recommend a total thyroidectomy and concomitant central lymph node dissection (CLND) in patients with clinically node-negative PTMC to avoid a reoperation ${ }^{13}$ or reduce the locoregional recurrence rate. ${ }^{14}$ However, elective CLND might increase the risk of postoperative complications, especially permanent hypocalcemia. ${ }^{15,16}$

The risk of recurrence in PTC can be estimated based on selected clinicopathologic features, such as the presence of extrathyroidal extension, aggressive histologies, vascular invasion, regional metastases, or high levels of postoperative serum thyroglobulin. The 2009 ATA guidelines for the management of thyroid cancer proposed a system to estimate the risk of relapse of PTC based on these clinicopathologic findings. ${ }^{17}$ Additional prognostic variables, such as the extent of lymph-node involvement and B-Raf proto-oncogene, serine/threonine kinase (BRAF) mutation profile were included in an updated version of the 2015 ATA risk stratification system $^{12}$ (-Table 1). However, these additional variables have not been rigorously assessed.

The aim of the present study was to review the characteristics of PTMC at diagnosis in retrospective cohort from a single cancer center, and to identify the clinical and pathological features associated with tumor recurrence. We also evaluated the 2009 ATA risk stratification system for the prediction of recurrence.

Table 1 Initial American Thyroid Association risk of recurrence classification

\begin{tabular}{|l|l|}
\hline $\begin{array}{l}\text { Low risk } \\
\text { (all of the following:) }\end{array}$ & No local or distant metastases; \\
& All macroscopic tumor has been resected; \\
& Tumor invasion of locoregional tissues; \\
& cell carcinoma, follicular thyroid cancer); \\
& No vascular invasion; \\
& No ${ }^{131}$ I uptake outside the thyroid bed on the posttreatment scan, if done; \\
& Clinical N0 or $\leq 5$ pathologic N1 micrometastases ( $<0.2 \mathrm{~cm})^{*}$ \\
& Intrathyroidal, encapsulated follicular variant of papillary thyroid cancer;* \\
& Intrathyroidal, well-differentiated follicular thyroid cancer with capsular invasion and no or minimal \\
& (<4 foci) vascular invasion; \\
& Intrathyroidal, papillary microcarcinoma, unifocal or multifocal, including BRAFV600E mutated (if \\
& known).* \\
\hline Intermediate risk \\
(any of the following:)
\end{tabular}

Note: *Additional prognostic variables included in the 2015 American Thyroid Association (ATA) risk stratification system. 


\section{Methods}

\section{Study Population and Treatment}

After obtaining approval form the institutional review board (ethics committee approval number: 2.904.573), we retrospectively reviewed the medical records of 4,085 consecutive patients treated for PTC between January 1996 and December 2015. Among these, we selected all 2,538 patients with PTMC. Only patients with a postoperative pathologic diagnosis of PTC and a maximum tumor diameter of $1 \mathrm{~cm}$ were included. Most patients had an initial total or subsequent completion thyroidectomy, which were performed at our institution based on patient preference and clinical criteria such as previous neck irradiation, hypothyroidism, familial predisposition or bilateral nodularity. Many physicians and patients chose bilateral thyroidectomies aiming to simplify the follow-up. Therapeutic lymph node dissection was performed if the clinical involvement was confirmed based on sonographic findings and intraoperative exploration of the central neck compartment. Elective CLND was performed in the presence of extrathyroidal extension. For patients who were pathologically confirmed to have high-risk findings, mainly extrathyroidal extension and cervical lymph node metastasis, routine radioactive iodine (RAI, or I-131) treatment was administered after withdrawal of hormone therapy for at least 4 weeks. Some patients were treated with RAI ablation, with the purpose of facilitating the follow-up or destroying the foci of micrometastatic disease. Diagnostic scintigraphy was performed before the administration of I131 and 2 to 5 days later. The levels of thyroglobulin (Tg), and anti-Tg antibodies were measured postoperatively just before the RAI treatment. Most PTMC patients received oral therapy with levothyroxine postoperatively, in an attempt to maintain their thyroid-stimulating hormone (TSH) levels below $2.0 \mathrm{mlU} / \mathrm{L}$.

\section{Follow-up}

The patients were assessed every 3 months for the first year, every 6 months between the second and fifth years, and every 12 months thereafter at the discretion of the attending physician, based on the risk of the individual patient. The follow-up visits included palpation of the neck, dosage of $\mathrm{TSH}, \mathrm{Tg}$ and anti-Tg antibody levels, and ultrasound examination of the cervical lymph nodes. Disease recurrence was defined as the first clinical reappearance of the tumor. It included all clinical events reported (local relapses, lymph node metastases, and distant metastases) and those confirmed by imaging modalities, biopsy or surgery.

\section{Prognostic Parameters}

Patient characteristics, surgery data, pathological features and postoperative clinical outcomes were retrieved from the medical charts. The pathological characteristics of the thyroidectomy specimens evaluated included: tumor size, extrathyroidal extension, multifocality, aggressive histological variant (such as tall cells, diffuse sclerosing and solid variants), neck lymph-node metastasis, lympho-vascular invasion, and chronic lymphocytic thyroiditis. The patients were classified according to the 2009 ATA risk stratification system as low, intermediate or high risk of recurrence ${ }^{17}$ ( - Table 1). Due to the small number of deaths, the overall survival was not analyzed.

\section{Statistical Methods}

The primary endpoint of the study was disease-free survival (DFS). The duration of the of follow-up was calculated as the interval between surgery and death or the last visit to the clinic. The categorical variables were described as the frequency of different categories, and the continuous variables, as means and standard deviations. The Kaplan-Meier method was used to evaluate the DFS. Comparisons between the categorical variables were initially performed using the logrank technique. The Cox univariate analysis was used to compare the survival analysis, and the significant variables were included in the multivariate Cox model. Values of $p<0.05$ were considered statistically significant. All analyses were performed using the Stata (StataCorp LP, College Station, TX, US).

\section{Results}

From January 1996 to December 2015, both the number of patients treated for papillary thyroid carcinoma (PTC) and the proportion of papillary thyroid microcarcinomas (PTMCs) increased: 42\% (29 out of 69 patients) from 1996 to $2000,60.8 \%$ (262 out of 431 ) from 2001 to $2005,61.9 \%$ (596 out of 963 ) from 2006 to 2010 , and $63 \%$ (1,651 out of 2,622) from 2011 to 2015 (-Fig. 1).

Among the 4,085 consecutive patients treated for PTC, we included 2,538 patients (62.1\%) with PTMC for the present retrospective study. Patients and tumor characteristics are presented in $\mathbf{- T a b l e} \mathbf{2}$.

Most PTMC $(2,292 ; 90.3 \%)$ were nonpalpable nodules incidentally diagnosed during neck radiologic procedures, such as ultrasonography or computed tomography, performed during follow-up due to other cancers or detected through the postoperative pathologic examination of surgical specimens resected for benign thyroid diseases or after prophylactic thyroidectomies.

Almost all patients underwent initial total thyroidectomy $(2,394 ; 94.3 \%)$ or completion thyroidectomy $(93 ; 3.7 \%)$. Central lymph-node dissection was performed in 212 (8.4\%) patients, 53 (2.1\%) of whom underwent concomitant lateral dissection. Radioactive iodine was adminidtered postsurgically in 1,311 (51.7\%) patients. The dose of iodine ranged from $30 \mathrm{mCi}$ to $425 \mathrm{mCi}$ (mean: $132.2 \mathrm{mCi}$ ).

After a mean follow-up of 58 months (range: 3 to 236.5 months), tumor recurrence was diagnosed in $63(2.5 \%)$ patients, mostly in the cervical lymph nodes (-Fig. 2). The median time to recurrence was of 58.1 months (range: 3 to 236.5 months; standard deviation: 40.4 ). There were no cancer-related deaths.

The log-rank univariate survival analysis showed that male gender $(p=0.003)$, age $<55$ years $(p=0.007)$, tumor size $>5 \mathrm{~mm}(p=0.008)$, multifocality $(p=0.001)$, and the presence of lymph-node metastases at diagnosis $(P<0.001)$ were significantly associated with tumor 


\section{PAPILLARY THYROID CARCINOMAS AND PROPORTION OF MICROCARCINOMAS}

Microcarcinomas Carcinomas $>1 \mathrm{~cm}$

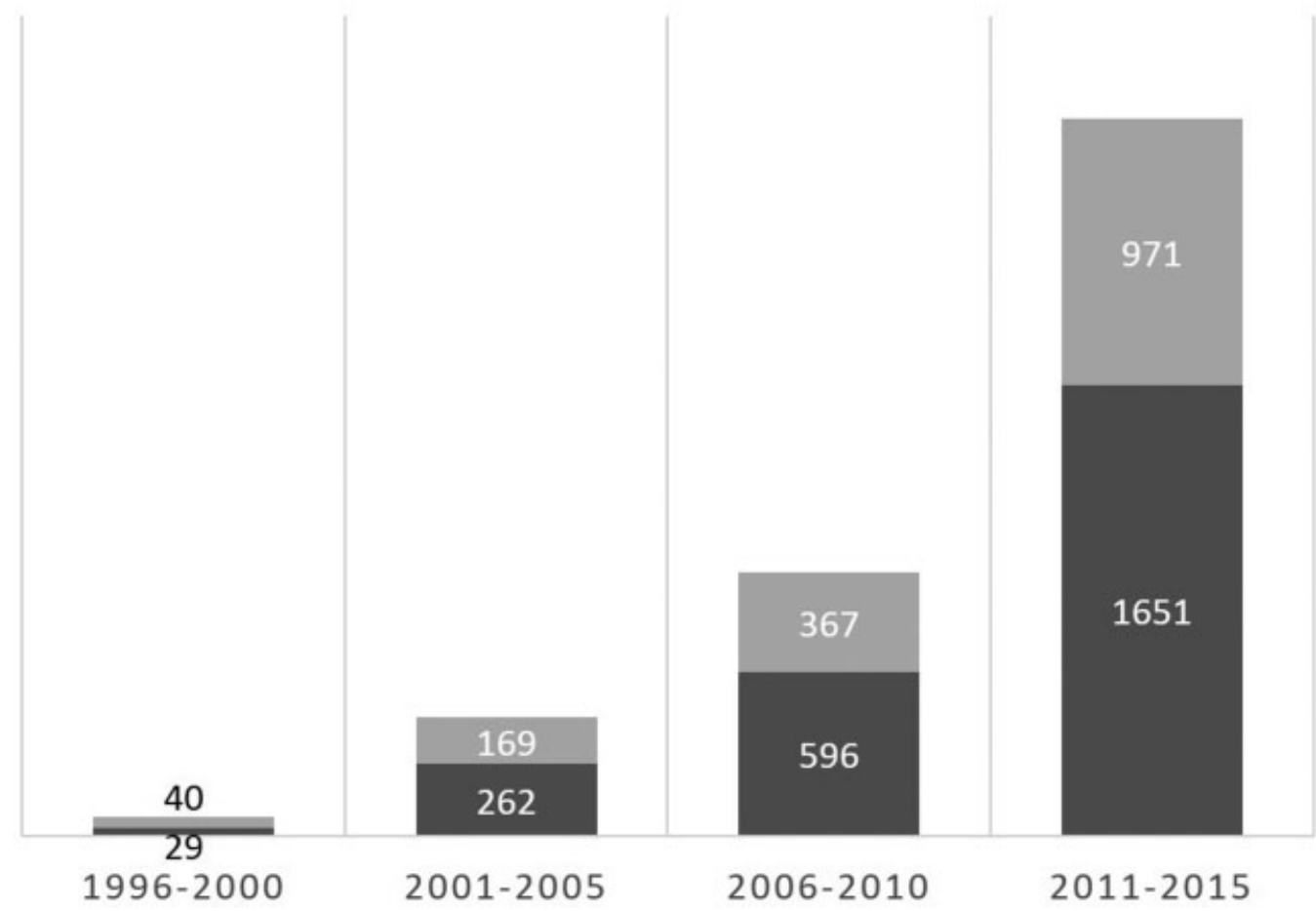

Fig. 1 Temporal evolution of papillary thyroid carcinomas and proportion of microcarcinomas.

Table 2 Patients and tumor characteristics

\begin{tabular}{|c|c|c|c|}
\hline Characteristics & & & Patients (N) \\
\hline \multirow[t]{2}{*}{ Gender } & Female & $83 \%$ & 2,106 \\
\hline & Male & $17 \%$ & 432 \\
\hline \multirow[t]{4}{*}{ Age (years): } & Mean (standard deviation) & $44.4(13.1)$ & 2,538 \\
\hline & Range & $7-85$ & \\
\hline & $<55$ & $78.3 \%$ & 1,696 \\
\hline & $\geq 55$ & $21.7 \%$ & 470 \\
\hline \multirow[t]{4}{*}{ Tumor size (mm): } & Mean (standard deviation) & $6.1(9.9)$ & \\
\hline & Range & $0.2-10$ & \\
\hline & $\leq 5 \mathrm{~mm}$ & $41 \%$ & 1,041 \\
\hline & $>5 \mathrm{~mm}$ & $59 \%$ & 1,497 \\
\hline Aggressive histology & & $3 \%$ & 75 \\
\hline Multifocality & & $30.2 \%$ & 766 \\
\hline \multirow[t]{2}{*}{ Extrathyroidal extension } & Minor & $14.1 \%$ & 357 \\
\hline & Gross & $0.2 \%$ & 7 \\
\hline Lympho-vascular invasion & & $1.2 \%$ & 30 \\
\hline Lymph-node metastasis & & $9.5 \%$ & 242 \\
\hline Chronic lymphocytic thyroiditis & & $32.7 \%$ & 830 \\
\hline \multirow[t]{3}{*}{ American Thyroid Association risk stratification category } & Low & $76.4 \%$ & 1,940 \\
\hline & Intermediate & $23.3 \%$ & 590 \\
\hline & High & $0.3 \%$ & 8 \\
\hline
\end{tabular}




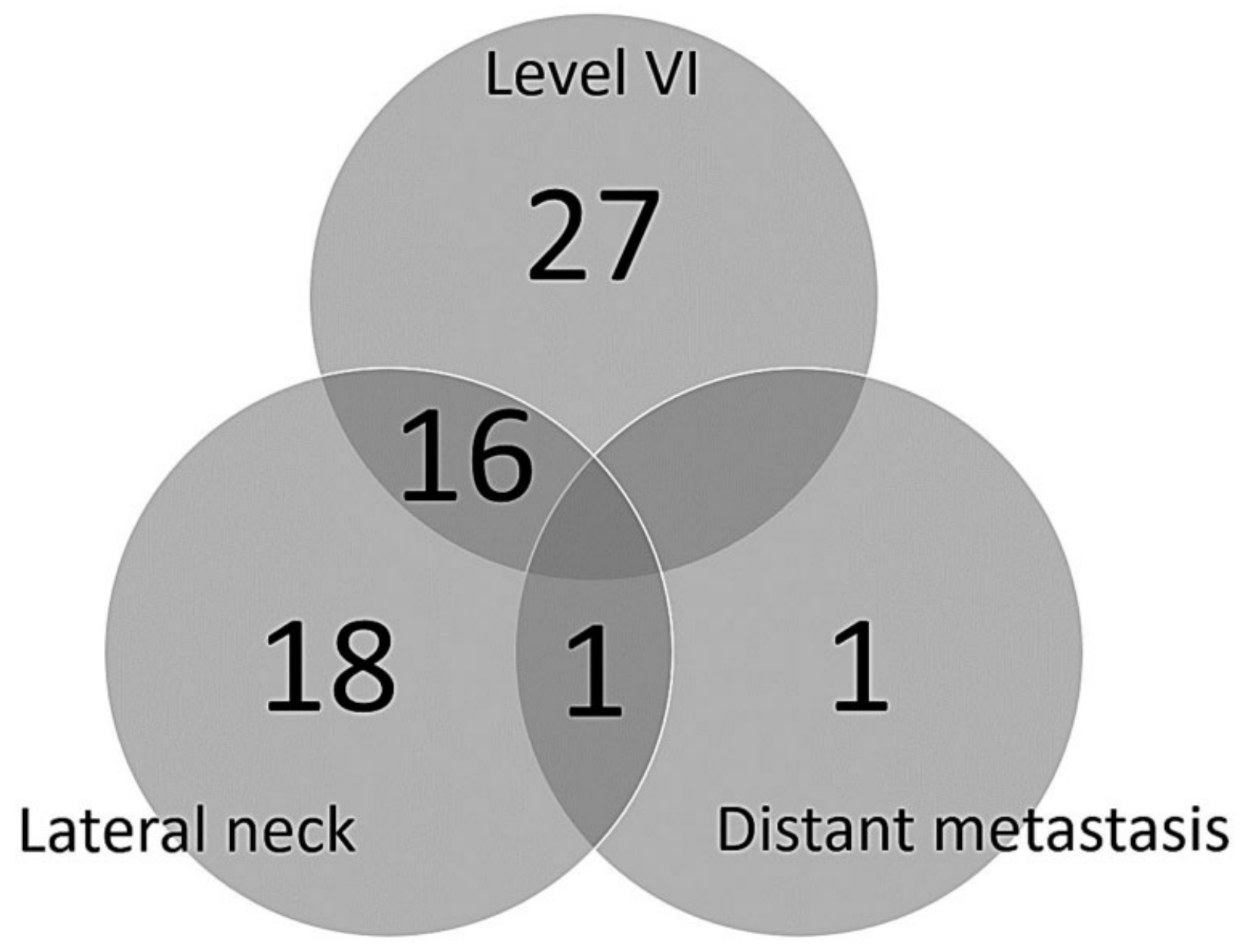

Fig. 2 Pattern of recurrence of papillary thyroid microcarcinoma.

recurrence. However, other pathological factors, such as extrathyroidal extension $(p=0.126)$, aggressive histological variants $(p=0.478)$, lympho-vascular invasion $(p=0.175)$, and chronic lymphocytic thyroiditis $(p=0.582)$ did not affect the DFS rate. Multivariate Cox regression analyses revealed that cancer recurrence was independently associated with age $<55$ years ( $p=0.049$; HR: 2.54 ; $95 \%$ CI: 0.95 to 0.99 ), multifocality ( $p=0.032$; HR: $1.76 ; 95 \%$ CI: 1.05 to 2.96$)$, and the presence of lymph-node metastasis ( $p<0.001$; HR: 3.69; 95\%CI: 2.07 to 6.57 ) (-Table 3).

Table 3 Univariate and multivariate cancer-recurrence logistic regression analyses of patients with papillary thyroid microcarcinoma

\begin{tabular}{|l|l|l|l|l|}
\hline \multirow{2}{*}{} & \multicolumn{2}{|l|}{ Univariate analysis } & \multicolumn{2}{l|}{ Multivariate analysis } \\
\cline { 2 - 5 } & $\begin{array}{l}\text { Hazard ratio (95\% confidence } \\
\text { interval) }\end{array}$ & $p$-value & $\begin{array}{l}\text { Hazard ratio (95\% confidence } \\
\text { interval) }\end{array}$ & $p$-value \\
\hline Male gender & $2.30(1.32-4.02)$ & 0.003 & $1.68(0.94-3.02)$ & 0.081 \\
\hline Age $<55$ years & $3.50(1.41-8.74)$ & 0.007 & $2.54(0.95-0.99)$ & 0.049 \\
\hline Tumor size $>5$ mm & $1.15(1.04-1.27)$ & 0.008 & $1.07(0.95-1.19)$ & 0.203 \\
\hline Multifocality & $2.33(1.42-3.83)$ & 0.001 & $1.76(1.05-2.96)$ & 0.032 \\
\hline Extrathyroidal extension & $1.61(0.87-2.98)$ & 0.126 & $0.88(0.45-1.71)$ & 0.706 \\
\hline Aggressive histology & $1.52(0.21-2.09)$ & 0.478 & $1.45(0.45-1.71)$ & 0.531 \\
\hline Lympho-vascular invasion & $2.05(0.725-5.86)$ & 0.175 & $1.22(0.43-3.51)$ & 0.708 \\
\hline Lymph-node metastasis & $5.56(3.31-9.34)$ & $<0.001$ & $3.69(2.07-6.57)$ & $<0.001$ \\
\hline Chronic thyroiditis & $0.86(0.51-1.46)$ & 0.582 & $0.98(0.57-1.67)$ & 0.936 \\
\hline
\end{tabular}




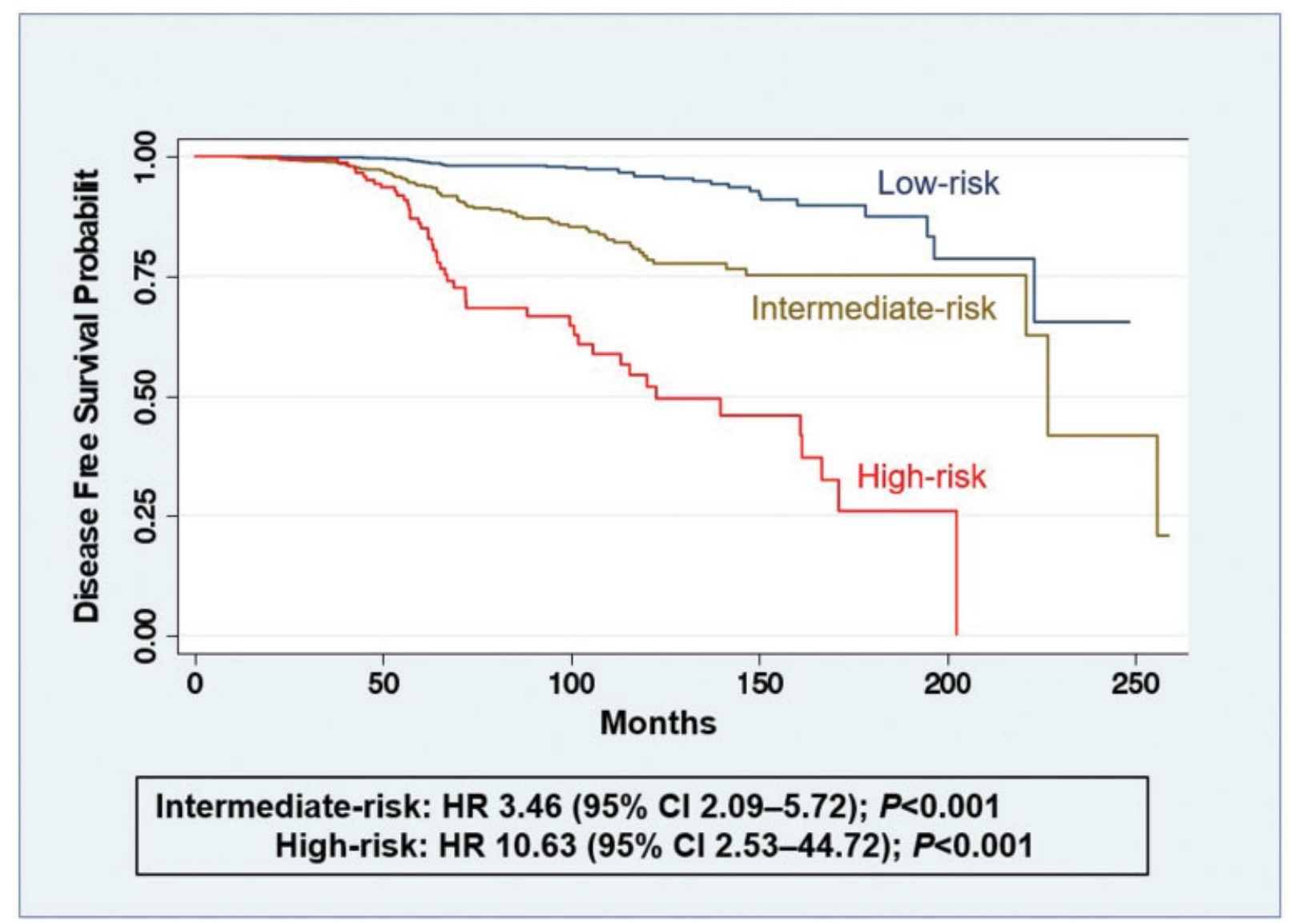

Fig. 3 Kaplan-Meier recurrence estimates based on American Thyroid Association risk categories.

According to the 2009 ATA risk stratification system, the patients were classified as low (1,940 - 76.4\%), intermediate (590 - 23.3\%) or high-risk (8 - 0.3\%).. Recurrence was observed in 29 (1.5\%) out of 1,940 low-risk patients, $32(5.4 \%)$ out of 590 intermediate-risk patients, and in 2 (25\%) out of 8 high-risk patients. The probability of 5-year DFS was significantly lower in high-risk (84.9\%) than in intermediate (94\%) and low-risk (98.8\%) patients (-Fig. 3 ).

\section{Discussion}

The incidence of PTMC is rising, mostly due to the increased presurgical diagnosis of incidental nonpalpable tumors. ${ }^{18}$ The prevalence of occult PTMC in autopsy specimens is high, ranging from $11.3 \%$ to $35.6 \%{ }^{19,20}$ depending on the extent of the histologic examination of the thyroid gland. A similar high frequency of incidental PTMCs is observed in $7.2 \%$ of thyroid glands surgically resected for benign diseases or after prophylactic thyroidectomies. ${ }^{21}$ In the present series, PTMCs were mainly incidental cancers detected during neck radiologic procedures or after postoperative pathologic examination of surgical specimens resected for benign thyroid diseases. As we know, incidental PTMC has different clinical characteristics and a much lower recurrence rate than non-incidental PTMC. ${ }^{22}$

The 2015 ATA guidelines recommend initial unilateral lobectomy for PTMC, with subsequent long-term surveillance. In properly selected low-risk patients, the extent of the initial thyroid surgery probably has little impact on disease-specific survival. While recurrence rates can be a little high in patients submitted to unilateral thyroidectomy, salvage therapy is quite effective in the few patients that have recurrence. Besides, the surgical risks of two-stage thyroidectomy (lobectomy followed by completion thyroidectomy) are similar to those of a neartotal or total thyroidectomy. ${ }^{12}$ Instead of unilateral resection, some investigators favor total thyroidectomy as an appropriate initial treatment for some cases of PTMC, with the advantages of providing lower local recurrence because of the removal of all potential foci in both lobes, improving the sensitivity of thyroglobulin as a tumor marker, and enabling the use of RAI in the detection of metastasis and recurrence during followup. $^{23-28}$ It is important to note that thyroid lobectomy is associated with an increased risk of recurrence, but not mortality, compared with total thyroidectomy for PTMC. ${ }^{29}$ In our institution, most patients were submitted to total thyroidectomy, based on patient preference and clinical criteria such as previous neck irradiation, hypothyroidism, familial predisposition, bilateral nodularity, or as a strategy to simplify the follow-up. Most authors, ${ }^{30,31}$ but not all, ${ }^{32,33}$ agree that postthyroidectomy RAI ablation is not beneficial in reducing cancer recurrence in PTMC patients. Otherwise, RAI ablation can make the follow-up easier by improving the sensitivity of Tg and anti-Tg antibodies. ${ }^{34}$

In general, recurrence of cancer is not frequently observed following thyroidectomy for PTMC. In a meta-analysis including 6,839 PTMC patients, Yi et al. ${ }^{35}$ found a low 
recurrence rate $(2.8 \%)$, very similar to ours (2.5\%). However, recurrence rates as high as $14.3 \%$ and $19 \%$ have been described in the literature. ${ }^{36,37}$ As expected, most of our patients had recurrence in the lymph nodes: $42.9 \%$ exclusively in level VI; $28.6 \%$ in the lateral neck levels; and $25.4 \%$ in both central and lateral compartment nodes. Recurrence is directly associated with the risk of regional spread. For PTMC patients, the incidence of occult lymph node metastasis is as high as $33 \%$, and these occur mainly in the central compartment of the neck. ${ }^{38}$ However, microscopic nodal disease is rarely of clinical importance, since it often remains quiescent or the subsequent RAI administration ablates these occult foci. Therefore, we do not routinely perform elective CLND in most PTMC patients. Meta-analyses revealed that lymphnode metastases in PTMC patients are associated with the male gender, younger age $(<45$ years), larger tumor size ( $>5 \mathrm{~mm}$ ), multifocality, extrathyroidal extension, and lymphovascular invasion, but not with thyroid bilaterality and chronic lymphocytic thyroiditis. ${ }^{38,39}$

Several clinicopathologic factors have been described to predict the recurrence of $\mathrm{PTMC}^{40}$ : tumor diameter $>5 \mathrm{~mm}$ or $7 \mathrm{~mm},{ }^{41,42}$ younger age, ${ }^{43-45}$ the male gender, ${ }^{42,43}$ multifocality, ${ }^{37,42,45,46}$ capsular invasion, ${ }^{47}$ or absence of tumor capsule, $^{37}$ extrathyroid extension, ${ }^{37,43,45,47,48}$ lymph node metastases, ${ }^{43,44,46-50}$ the CLNM ratio (number of metastasized and removed nodes at the first operation) $>0.5,41,51$ aggressive histological variants, ${ }^{37,52}$ mutated $\mathrm{BRAF}^{53}$ and non-incidental diagnosis. ${ }^{54}$ Our data showed that younger age ( $<55$ years), multifocality, and presence of lymph node metastases were independently associated with tumor recurrence in PTMC patients. Additionally, the male gender and maximum tumor diameter $>5 \mathrm{~mm}$ also increased the risk of recurrence on the univariate analysis. In our cohort, most PTMCs (14.1\%) presented minor extrathyroidal extension compared with only $0.2 \%$ of those with gross extension to the perithyroidal tissues. Similarly, some authors previously showed that gross but not minimal extrathyroidal extension is a significant factor associated with tumor recurrence in PTC patients. ${ }^{55-57}$ Other pathological factors, such as aggressive histology and lympho-vascular invasion, did not affect the DFS rate, probably due the small number of cases. Finely, chronic lymphocytic thyroiditis was found in one third of PTMC patients, with no impact on cancer recurrence. Actually, previous publications found that lymphocytic thyroiditis resulted in a decreased risk of lymphnode metastases. ${ }^{58,59}$

Although PTMC is generally associated with an excellent prognosis, some patients will experience poor outcomes. An analysis of 46,662 patients with PTMC from the Surveillance, Epidemiology, and End Results (SEER) program (1983-2015) showed 5-year, 10-year, and 20-year probabilities of death of $0.3 \%, 0.6 \%$, and $1.4 \%$ respectively. Older age at diagnosis, male gender, tumor extension and lymph-node involvement were related to the cumulative incidence of death. ${ }^{60}$ Metastatic PTMCs have been described in the literature. The location of the distant metastases was primarily pulmonary, and most patients, ${ }^{47,61}$ but not all, ${ }^{62}$ had lymph node involvement on the initial presentation. In our series, two patients presented metastases in the lungs and bones, with no cancer-related deaths.

The 2017 tumor, node, metastasis (TNM) staging system of the American Joint Cancer Committee/Union for International Cancer Control (AJCC/UICC) is adequately used to predict disease-specific mortality. ${ }^{63}$ Since death is uncommon following the management of PTMC patients, we also use the ATA clinicopathologic staging system to provide initial estimates of the risk of recurrence and thus improve clinical decision-making. ${ }^{64}$ As expected, most of our PTMC patients were classified as 2009 ATA low-risk (76.4\%) or intermediate-risk of relapse (23.3\%). The risk of recurrence in PTMC patients was very low and effectively predicted by the ATA staging system. Moreover, while the TNM and ATA staging systems can be used to guide the initial therapeutic and diagnostic strategy decisions, it is important to recognize that initial risk estimates may be refined as new information is accumulated during the first two years of follow-up monitoring. ${ }^{65}$ As an example, in patients with successfully-treated PTC (postoperative undetectable s-Tg levels and no evidence of disease on whole-body iodine scan after total thyroidectomy and RAI ablation), recurrence-free survival did not differ between patients classified as high-risk and those classified as low-risk based on TNM stage at diagnosis. ${ }^{66}$ Thus, further prospective studies are required to investigate the impact of this dynamic risk assessment on ATA initial-risk estimates.

Some limitations of this retrospective study are mainly related to selection bias. Recommendations on treatment and on the intensity and frequency of follow-up visits and tests varied from patient to patient, based on individual surgeons and patient preferences, and not on an institutional protocol. This would lead to an increase in the diagnosis of recurrent disease in intermediate- to high-risk patients in comparison with the less rigorous testing paradigm often used in low-risk patients. Furthermore, important prognostic variables included in the updated version of the 2015 ATA risk stratification system, such as the number and dimension of lymph-node metastases, were not assessed in the present study. Finally, a median follow-up period of 58 months may be short, as some patients with a less aggressive disease may manifest clinically-significant recurrence many years following the initial therapy.

\section{Conclusion}

In summary, our data confirm that clinical and pathological factors such as age $<55$ years, multifocality, and the presence of lymph-node metastasis at diagnosis are good initial predictors of recurrence in PTMC patients. Further, our data confirm that the ATA recurrence staging system effectively predicts recurrence, thus providing valuable information that can help individualize the clinical management and follow-up for PTMC patients.

\section{Conflict of Interests}

The authors have no conflict of interests to declare. 


\section{References}

1 Wiltshire JJ, Drake TM, Uttley L, Balasubramanian SP. Systematic Review of Trends in the Incidence Rates of Thyroid Cancer. Thyroid 2016;26(11):1541-1552

2 Roti E, degli Uberti EC, Bondanelli M, Braverman LE. Thyroid papillary microcarcinoma: a descriptive and meta-analysis study. Eur J Endocrinol 2008;159(06):659-673

3 Londero SC, Krogdahl A, Bastholt L, et al;Danish Thyroid Cancer Group. Papillary thyroid microcarcinoma in Denmark 1996-2008: a national study of epidemiology and clinical significance. Thyroid 2013;23(09):1159-1164

4 Reinke R, Mathiesen JS, Larsen SR, et al;A study from The Danish Thyroid Cancer Group - DATHYRCA (part of the DAHANCA organization) Incidental and Non-incidental Papillary Thyroid Microcarcinoma in Denmark 1996-2015: A national study on incidence, outcome and thoughts on active surveillance. Cancer Epidemiol 2019;60:46-50

5 Hedinger C, Williams E, Sobin L. 1988Histologic types of thyroid tumors in World Health Organization histological classification of tumors. 2nd ed. Verlag: Springer; 9-10

6 Zheng W, Wang X, Rui Z, Wang Y, Meng Z, Wang R. Clinical features and therapeutic outcomes of patients with papillary thyroid microcarcinomas and larger tumors. Nucl Med Commun 2019;40(05):477-483

7 Hay ID, Grant CS, van Heerden JA, et al. Papillary thyroid microcarcinoma: a study of 535 cases observed in a 50 -year period. Surgery 1992;112:1139Y1146

8 Noguchi S, Yamashita H, Murakami N, et al. Small carcinomas of the thyroid a long-term follow-up of 867 patients. Arch Surg 1996;131(02):187-191

9 Nabhan F, Ringel MD. Thyroid nodules and cancer management guidelines: comparisons and controversies. Endocr Relat Cancer 2017;24(02):R13-R26

10 Ito Y, Miyauchi A, Oda H. Low-risk papillary microcarcinoma of the thyroid: A review of active surveillance trials. Eur J Surg Oncol 2018;44(03):307-315

11 Miyauchi A, Ito Y, Oda H. Insights into the Management of Papillary Microcarcinoma of the Thyroid. Thyroid 2018;28(01):23-31

12 Haugen BR, Alexander EK, Bible KC, et al. 2015 American Thyroid Association Management Guidelines for Adult Patients with Thyroid Nodules and Differentiated Thyroid Cancer: The American Thyroid Association Guidelines Task Force on Thyroid Nodules and Differentiated Thyroid Cancer. Thyroid 2016;26(01):1-133

13 Shi L, Chen JH, Wang ST, Xiong YQ, Huang T. Treatment for papillary thyroid microcarcinoma. Contemp Oncol (Pozn) 2013; 17(01):20-23

14 Su H, Li Y. Prophylactic central neck dissection and local recurrence in papillary thyroid microcarcinoma: a meta-analysis. Rev Bras Otorrinolaringol (Engl Ed) 2019;85(02):237-243

15 So YK, Seo MY, Son YI. Prophylactic central lymph node dissection for clinically node-negative papillary thyroid microcarcinoma: influence on serum thyroglobulin level, recurrence rate, and postoperative complications. Surgery 2012;151(02):192-198

16 Ywata de Carvalho A, Chulam TC, Kowalski LP. Long-term Results of Observation vs Prophylactic Selective Level VI Neck Dissection for Papillary Thyroid Carcinoma at a Cancer Center. JAMA Otolaryngol Head Neck Surg 2015;141(07):599-606

17 Cooper DS, Doherty GM, Haugen BR, et al;American Thyroid Association (ATA) Guidelines Taskforce on Thyroid Nodules and Differentiated Thyroid Cancer. Revised American Thyroid Association management guidelines for patients with thyroid nodules and differentiated thyroid cancer. Thyroid 2009;19(11): 1167-1214

18 Kaliszewski K, Zubkiewicz-Kucharska A, Kiełb P, Maksymowicz J, Krawczyk A, Krawiec O. Comparison of the prevalence of incidental and non-incidental papillary thyroid microcarcinoma during 20082016: a single-center experience. World J Surg Oncol 2018;16(01):202
19 Yamamoto Y, Maeda T, Izumi K, Otsuka H. Occult papillary carcinoma of the thyroid. A study of 408 autopsy cases. Cancer 1990;65(05):1173-1179

20 Harach HR, Franssila KO, Wasenius VM. Occult papillary carcinoma of the thyroid. A "normal" finding in Finland. A systematic autopsy study. Cancer 1985;56(03):531-538

21 de Matos PS, Ferreira AP, Ward LS. Prevalence of papillary microcarcinoma of the thyroid in Brazilian autopsy and surgical series. Endocr Pathol 2006;17(02):165-173

22 Mehanna H, Al-Maqbili T, Carter B, et al. Differences in the recurrence and mortality outcomes rates of incidental and nonincidental papillary thyroid microcarcinoma: a systematic review and meta-analysis of 21329 person-years of follow-up. J Clin Endocrinol Metab 2014;99(08):2834-2843

23 Karatzas T, Vasileiadis I, Kapetanakis S, Karakostas E, Chrousos G, Kouraklis G. Risk factors contributing to the difference in prognosis for papillary versus micropapillary thyroid carcinoma. Am J Surg 2013;206(04):586-593

24 Hay ID, Hutchinson ME, Gonzalez-Losada T, et al. Papillary thyroid microcarcinoma: a study of 900 cases observed in a 60-year period. Surgery 2008;144(06):980-987, discussion 987-988

25 Ito Y, Miyauchi A, Jikuzono T, et al. Risk factors contributing to a poor prognosis of papillary thyroid carcinoma: validity of UICC/AJCC TNM classification and stage grouping. World J Surg 2007;31(04):838-848

26 Cappelli C, Castellano M, Braga M, et al. Aggressiveness and outcome of papillary thyroid carcinoma (PTC) versus microcarcinoma (PMC): a mono-institutional experience. J Surg Oncol 2007; 95(07):555-560

27 Giordano D, Gradoni P, Oretti G, Molina E, Ferri T. Treatment and prognostic factors of papillary thyroid microcarcinoma. Clin Otolaryngol 2010;35(02):118-124

28 Ross DS, Litofsky D, Ain KB, et al. Recurrence after treatment of micropapillary thyroid cancer. Thyroid 2009;19(10):1043-1048

29 Zheng W, Li J, Lv P, Chen Z, Fan P. Treatment efficacy between total thyroidectomy and lobectomy for patients with papillary thyroid microcarcinoma: A systemic review and meta-analysis. Eur J Surg Oncol 2018;44(11):1679-1684

30 Hu G, Zhu W, Yang W, Wang H, Shen L, Zhang H. The Effectiveness of Radioactive Iodine Remnant Ablation for Papillary Thyroid Microcarcinoma: A Systematic Review and Meta-analysis. World J Surg 2016;40(01):100-109

31 Kwon H, Jeon MJ, Kim WG, et al. Lack of Efficacy of Radioiodine Remnant Ablation for Papillary Thyroid Microcarcinoma: Verification Using Inverse Probability of Treatment Weighting. Ann Surg Oncol 2017;24(09):2596-2602

32 Yang T, Zheng SY, Jiao J, Zou Q, Zhang Y. Radioiodine remnant ablation in papillary thyroid microcarcinoma: a meta-analysis. Nucl Med Commun 2019;40(07):711-719

33 Mihailovic J, Stefanovic L, Stankovic R. Influence of initial treatment on the survival and recurrence in patients with differentiated thyroid microcarcinoma. Clin Nucl Med 2013;38(05):332-338

34 Soydal C, Araz M, Ozkan E, Arslantaş E, Kucuk ON, Aras G. Assessment of recurrence rates in papillary thyroid microcarcinoma patients with and without histopathological risk factors after radioiodine ablation treatment. Nucl Med Commun 2015;36 (02):109-113

35 Yi D, Song P, Huang T, Tang X, Sang J. A meta-analysis on the effect of operation modes on the recurrence of papillary thyroid microcarcinoma. Oncotarget 2017;8(04):7148-7156

36 Gao X, Zhang X, Zhang Y, Hua W, Maimaiti Y, Gao Z. Is papillary thyroid microcarcinoma an indolent tumor?: A retrospective study on 280 cases treated with radioiodine Medicine (Baltimore) 2016;95(40):e5067

37 Ardito G, Revelli L, Giustozzi E, et al. Aggressive papillary thyroid microcarcinoma: prognostic factors and therapeutic strategy. Clin Nucl Med 2013;38(01):25-28 
38 Qu N, Zhang L, Ji QH, et al. Risk Factors for Central Compartment Lymph Node Metastasis in Papillary Thyroid Microcarcinoma: A Meta-Analysis. World J Surg 2015;39(10):2459-2470

39 Liu LS, Liang J, Li JH, et al. The incidence and risk factors for central lymph node metastasis in cNO papillary thyroid microcarcinoma: a meta-analysis. Eur Arch Otorhinolaryngol 2017;274(03):1327-1338

40 Ruggiero R, Pirozzi R, Gualtieri G, et al. Overview on surgical management of papillary thyroid microcarcinoma. G Chir 2019; 40(02):81-87

41 Wang X, Lei J, Wei T, Zhu J, Li Z. Clinicopathological characteristics and recurrence risk of papillary thyroid microcarcinoma in the elderly. Cancer Manag Res 2019;11:2371-2377

$42 \mathrm{Xu} \mathrm{Y,} \mathrm{Xu} \mathrm{L,} \mathrm{Wang} \mathrm{J.} \mathrm{Clinical} \mathrm{predictors} \mathrm{of} \mathrm{lymph} \mathrm{node} \mathrm{metastasis}$ and survival rate in papillary thyroid microcarcinoma: analysis of 3607 patients at a single institution. J Surg Res 2018;221:128-134

43 Yu XM, Wan Y, Sippel RS, Chen H. Should all papillary thyroid microcarcinomas be aggressively treated? An analysis of 18,445 cases. Ann Surg 2011;254(04):653-660

44 Pedrazzini L, Baroli A, Marzoli L, Guglielmi R, Papini E. Cancer recurrence in papillary thyroid microcarcinoma: a multivariate analysis on 231 patients with a 12-year follow-up. Minerva Endocrinol 2013;38(03):269-279

45 Siddiqui S, White MG, Antic T, et al. Clinical and Pathologic Predictors of Lymph Node Metastasis and Recurrence in Papillary Thyroid Microcarcinoma. Thyroid 2016;26(06):807-815

46 Chow SM, Law SC, Chan JK, Au SK, Yau S, Lau WH. Papillary microcarcinoma of the thyroid-Prognostic significance of lymph node metastasis and multifocality. Cancer 2003;98(01):31-40

47 Mercante G, Frasoldati A, Pedroni C, et al. Prognostic factors affecting neck lymph node recurrence and distant metastasis in papillary microcarcinoma of the thyroid: results of a study in 445 patients. Thyroid 2009;19(07):707-716

48 Chéreau N, Buffet C, Trésallet C, et al. Does extracapsular extension impact the prognosis of papillary thyroid microcarcinoma? Ann Surg Oncol 2014;21(05):1659-1664

49 Baek SK, Jung KY, Kang SM, et al. Clinical risk factors associated with cervical lymph node recurrence in papillary thyroid carcinoma. Thyroid 2010;20(02):147-152

50 Lee J, Song Y, Soh EY. Central lymph node metastasis is an important prognostic factor in patients with papillary thyroid microcarcinoma. J Korean Med Sci 2014;29(01):48-52

51 Lombardi CP, Bellantone R, De Crea C, et al. Papillary thyroid microcarcinoma: extrathyroidal extension, lymph node metastases, and risk factors for recurrence in a high prevalence of goiter area. World J Surg 2010;34(06):1214-1221

52 Ghossein R, Ganly I, Biagini A, Robenshtok E, Rivera M, Tuttle RM. Prognostic factors in papillary microcarcinoma with emphasis on histologic subtyping: a clinicopathologic study of 148 cases. Thyroid 2014;24(02):245-253
53 Chen Y, Sadow PM, Suh H, et al. BRAF(V600E) Is Correlated with Recurrence of Papillary Thyroid Microcarcinoma: A Systematic Review, Multi-Institutional Primary Data Analysis, and MetaAnalysis. Thyroid 2016;26(02):248-255

54 Lo CY, Chan WF, Lang BH, Lam KY, Wan KY. Papillary microcarcinoma: is there any difference between clinically overt and occult tumors? World J Surg 2006;30(05):759-766

55 Radowsky JS, Howard RS, Burch HB, Stojadinovic A. Impact of degree of extrathyroidal extension of disease on papillary thyroid cancer outcome. Thyroid 2014;24(02):241-244

56 Jin BJ, Kim MK, Ji YB, Song CM, Park JH, Tae K. Characteristics and significance of minimal and maximal extrathyroidal extension in papillary thyroid carcinoma. Oral Oncol 2015;51(08):759-763

57 Shin JH, Ha TK, Park HK, et al. Implication of minimal extrathyroidal extension as a prognostic factor in papillary thyroid carcinoma. Int J Surg 2013;11(09):944-947

$58 \mathrm{Qu} \mathrm{H}$, Sun GR, Liu Y, He QS. Clinical risk factors for central lymph node metastasis in papillary thyroid carcinoma: a systematic review and meta-analysis. Clin Endocrinol (Oxf) 2015;83(01): 124-132

59 Vita R, Ieni A, Tuccari G, Benvenga S. The increasing prevalence of chronic lymphocytic thyroiditis in papillary microcarcinoma. Rev Endocr Metab Disord 2018;19(04):301-309

60 Wang K, Xu J, Li S, Liu S, Zhang L. Population-based study evaluating and predicting the probability of death resulting from thyroid cancer among patients with papillary thyroid microcarcinoma. Cancer Med 2019;8(16):6977-6985

61 Baudin E, Travagli JP, Ropers J, et al. Microcarcinoma of the thyroid gland: the Gustave-Roussy Institute experience. Cancer 1998;83 (03):553-559

62 Godbert Y, Henriques-Figueiredo B, Cazeau AL, et al. A papillary thyroid microcarcinoma revealed by a single bone lesion with no poor prognostic factors. Case Rep Endocrinol 2013;2013:719304

63 Tuttle RM, Morris LF, Haughen BR, et al. Thyroid - Differentiated and Anaplastic Carcinoma. In: AJCC Cancer Staging Manual, 8th, Amin MB (Ed). SpringerNew York2017:873

64 Stefanova DI, Bose A, Ullmann TM, et al. Does the ATA Risk Stratification Apply to Patients with Papillary Thyroid Microcarcinoma? World J Surg 2020;44(02):452-460

65 Tuttle RM, Tala H, Shah J, et al. Estimating risk of recurrence in differentiated thyroid cancer after total thyroidectomy and radioactive iodine remnant ablation: using response to therapy variables to modify the initial risk estimates predicted by the new American Thyroid Association staging system. Thyroid 2010;20 (12):1341-1349

66 Verburg FA, Stokkel MP, Düren C, et al. No survival difference after successful (131)I ablation between patients with initially low-risk and high-risk differentiated thyroid cancer. Eur J Nucl Med Mol Imaging 2010;37(02):276-283 